INPLASY

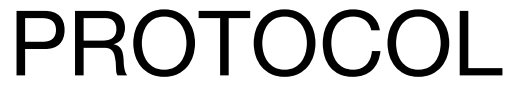

To cite: Chen et al. Qianzheng powder for the treatment of primary Hemifacial spasm. Inplasy protocol 202130008. doi:

10.37766/inplasy2021.3.0008

Received: 02 March 2021

Published: 03 March 2021

Corresponding author:

Baoshan Chen

1164925816@qq.com

Author Affiliation:

Jiangxi University of

Traditional Chinese Medicine, Nanchang, China

Support: Jiangxi province.

Review Stage at time of this submission: The review has not yet started.

Conflicts of interest:

None declared.

\section{Qianzheng powder for the treatment of primary Hemifacial spasm}

Chen, B'; Rigun, A2; Yue, R³; Zhang, G4.

Review question / Objective: Can Qianzheng powder improve quality of life in patients with Hemifacial spasm?

Condition being studied: Hemifacial spasm (HFS) is a clinical common neurological disease, its main performance for one side or two sides muscles (the orbicularis oculi muscle, expression, orbicularisoris muscles) recurrent paroxysmal, involuntary twitching, aggravating when excited or nervous, more severe cases of the disease may include difficulty in opening the eyes, crooked corners of the mouth, and twitching noises in the ears, etc.[1] The disease will affect the quality of life such as speaking, eating, seeing and so on, and even cause psychological effects such as inferiority, anxiety and depression. At present, the incidence of the disease in China is $\mathbf{1 1}$ per $\mathbf{1 . 1}$ million, females are more common than males. There are many ways to treat HFS, but the Qianzheng powder has a unique advantage in treating this disease. Therefore, our systematic review aims to evaluate the efficacy and safety of Qianzheng powder in the treatment of Primary Hemifacial spasm, and to provide a reliable basis for clinical decision makers.

INPLASY registration number: This protocol was registered with the International Platform of Registered Systematic Review and Meta-Analysis Protocols (INPLASY) on 03 March 2021 and was last updated on 03 March 2021 (registration number INPLASY202130008).

\section{INTRODUCTION}

Review question / Objective: Can Qianzheng powder improve quality of life in patients with Hemifacial spasm?
Condition being studied: Hemifacial spasm (HFS) is a clinical common neurological disease, its main performance for one side or two sides muscles (the orbicularis oculi muscle, expression, orbicularisoris muscles) recurrent paroxysmal, involuntary 
twitching, aggravating when excited or nervous, more severe cases of the disease may include difficulty in opening the eyes, crooked corners of the mouth, and twitching noises in the ears, etc.[1] The disease will affect the quality of life such as speaking, eating, seeing and so on, and even cause psychological effects such as inferiority, anxiety and depression. At present, the incidence of the disease in China is 11 per 1.1 million, females are more common than males.There are many ways to treat HFS, but the Qianzheng powder has a unique advantage in treating this disease. Therefore, our systematic review aims to evaluate the efficacy and safety of Qianzheng powder in the treatment of Primary Hemifacial spasm, and to provide a reliable basis for clinical decision makers.

\section{METHODS}

Participant or population: Participants are patients with Hemifacial spasm.

Intervention: Qianzheng powder is an intervention which can effectively improve the spastic intensity of patients with Hemifacial spasm.

Comparator: Participants without Qianzheng power.

Study designs to be included: Randomized controlled trials.

Eligibility criteria: Randomised controlled trials evaluating Qianzheng powder in the treatment of primary HFS will be eligible for inclusion and will be published in English or Chinese, with the full-text available.Patients with primary HFS with a clear diagnostic criteria will be included.

Information sources: We will search electronic databases, including PubMed, Embase, Cochrane Library, China Biomedical Literature Database (CBM), China National Knowledge Infrastructure (CNKI), Wanfang Database (WF), and China Science Journals Database (VIP), to collect potential RCTs from the start through April
2021.If there are any problems with the study, we will try to contact the author.

Main outcome(s): Total effective rate (assessed based on Cohen criteria for efficacy evaluation of hemifacial spasm, total effective rate $=($ cure + significant effect + effective)/total).

Quality assessment / Risk of bias analysis: The bias risk assessment tool recommended by the Cochrane Collaboration Network was used to assess the quality of the included studies. The following seven evaluation items are included: (1)random sequence generation; (2)allocation concealment; (3)blinding of participants and personnel; (4)blinding of outcome assessment; (5)incomplete outcome data; (6)selective reporting; (7)other sources of bias For each study, the results of the seven items were rated as "Yes" (low risk), "No" (high risk) and "unclear" (lack of information or uncertainty about bias). Two reviewers independently conduct quality assessments and any differences will be resolved through discussion.

Strategy of data synthesis: We will use RevMan V. 5.3 software for statistical analysis. For dichotomous variables, relative risk (RR) and $95 \%$ confidence intervals $(\mathrm{Cl})$ were used. We will use the $\mathrm{I}^{2}$ test and the Chi-square test to evaluate the heterogeneity of the results. When $\mathrm{I}^{2} 0.10$, the results will be considered homogeneous and the fixed-effect model will be used. Otherwise, the random effects model is used.

Subgroup analysis: If significant heterogeneity is detected in our metaanalysis, we will perform subgroup analysis according to different control groups.

Sensitivity analysis: When sufficient RCTs are available, we will conduct sensitivity analysis based on method quality, sample size, and missing data to assess the robustness of the study results.

Language: Chinese and English. 
Country(ies) involved: China.

Keywords: Primary Hemifacial spasm, Qianzheng powder, protocol, systematic review.

Contributions of each author:

Author 1 - Baoshan Chen.

Email: 1164925816@qq.com

Author 2 - Rigun A.

Email: 389014066@qq.com

Author 3 - Ruizhen Yue.

Email: 958499575@qq.com

Author 4 - Guangrong Zhang.

Email: zhgr321@163.com 